\title{
ATIVIDADE ANTIBACTERIANA DO EXTRATO AQUOSO DE Tripodanthus acutifolius FRENTE A Staphylococcus aureus
}

\author{
Thamires Klein de Souza $a^{1}$ \\ Chana de Medeiros da Silva ${ }^{2}$ \\ Lisianne Brittes Benitez ${ }^{3}$ \\ Gilsane de Lino von Poser ${ }^{4}$ \\ José Ângelo Silveira Zuanazzi ${ }^{5}$
}

\section{RESUMO}

As propriedades antimicrobianas de substâncias e óleos essenciais que as plantas contêm, como produtos de seu metabolismo secundário, têm sido alvo da comunidade científica, que se dedica ao estudo das propriedades medicinais das plantas. Considerando que em vários grupos de microrganismos é crescente o número de cepas resistentes aos antibióticos utilizados no tratamento de infecções, este estudo teve por objetivo avaliar o potencial antimicrobiano do extrato aquoso de Tripodanthus acutifolius (erva-de-passarinho) isolado do hospedeiro Ligustrum lucidum frente a cepas de Staphylococcus aureus com perfis de resistência (MRSA) ou sensibilidade à meticilina (MSSA). A atividade antimicrobiana foi testada pelo método de ágar-difusão em disco. Os resultados obtidos mostraram que o extrato aquoso de Tripodanthus acutifolius em concentrações que variaram de $100 \mathrm{mg} / \mathrm{mL}$ a 0,5 $\mathrm{mg} / \mathrm{mL}$ apresenta potencial antibacteriano contra Staphylococcus aureus evidenciado pelo aparecimento de zonas de inibição do crescimento. A atividade antibacteriana observada neste estudo sugere que o extrato aquoso de Tripodanthus acutifolius é fonte de compostos com atividade farmacológica e significativo potencial terapêutico.

Palavras-chave: Tripodanthus acutifolius. Extrato aquoso. Atividade antibacteriana. Staphylococcus aureus.

\begin{abstract}
The antimicrobial properties of substances and essential oils that plants contain as products of secondary metabolism has been targeted by the scientific community that is dedicated to studying the medicinal properties of plants. Considering that in several groups of microorganisms it is increased the number of strains resistant to the antibiotics used to treat infections, the aim of this study was to evaluate the antimicrobial activity of the Tripodanthus acutifolius (mistletoe) extract isolated from the host Ligustrum lucidum against strains of Staphylococcus aureus that had resistant (MRSA) profiles and sensitivity to methicillin (MSSA). Antimicrobial activity was tested by the method of agar disk diffusion. The results obtained by agar diffusion showed that the aqueous extract of Tripodanthus acutifolius in concentrations of $100 \mathrm{mg} / \mathrm{mL}$ to $0,5 \mathrm{mg} / \mathrm{mL}$ has antimicrobial activity against Staphylococcus aureus, evidenced by the appearance of zones of growth inhibition. The antibacterial activity

1 Acadêmica do Curso de Ciências Biológicas, Departamento de Biologia e Farmácia, Bolsista (PROBIC/FAPERGS), Universidade de Santa Cruz do Sul - UNISC. <thamireskleinbio@ yahoo.com.br>

2 Professora e Pesquisadora do Departamento de Biologia e Farmácia, Universidade de Santa Cruz do Sul UNISC. Doutoranda do Programa de Pós-graduação em Ciências Farmacêuticas, Universidade Federal do Rio Grande do Sul - UFRGS. <csilva@unisc.br>

${ }^{3}$ Professora e Pesquisadora do Departamento de Biologia e Farmácia e do Programa de Pós-Graduação Mestrado em Tecnologia Ambiental, Universidade de Santa Cruz do Sul - UNISC. <lisianne@ unisc.br>

${ }^{4}$ Professora e Pesquisadora do Programa de Pós-graduação em Ciências Farmacêuticas, Universidade Federal do Rio Grande do Sul - UFRGS. <gilsane@ farmacia.ufrgs.br>

${ }^{5}$ Professor e Pesquisador do Programa de Pós-graduação em Ciências Farmacêuticas, Universidade Federal do Rio Grande do Sul - UFRGS. <zuanazzi@ufrgs.br>
\end{abstract}


observed in this study suggests that the aqueous extract of Tripodanthus acutifolius is a source of compounds with significant pharmacological activity and therapeutic potential.

Keywords: Tripodanthus acutifolius. Aqueous extract. Antibacterial activity. Staphylococcus aureus.

\section{INTRODUÇÃO}

Há muitos anos, a população vem aprendendo sobre o benefício de utilizar plantas para amenizar ou curar doenças. O reino vegetal possui uma fonte de compostos químicos novos que podem ser importantes devido ao seu uso na medicina (SOBERÓN et al., 2007).

A atividade biológica das plantas medicinais tem sido frequente alvo de investigação científica, seja pelas inúmeras espécies vegetais, seja pelo comprovado espectro de atividade inibitória sobre fungos e bactérias. A ação inibitória dos vegetais superiores sobre microrganismos é conferida pela atividade metabólica secundária, que os torna aptos a produzirem substâncias antibióticas, usadas como mecanismo de proteção contra microrganismos, insetos e herbívoros (NUNES, 2008).

As plantas hemiparasitas crescem sobre os ramos de outros vegetais, dos quais retiram água e nutrientes minerais por meio de um sistema haustorial, mantendo, entretanto, sua capacidade fotossintética por possuir folhas normais providas de estômatos e clorofila, através da metabolização de substâncias orgânicas para o seu desenvolvimento (ROTTA, 2004).

O termo erva-de-passarinho é empregado devido à maioria das espécies dependerem de aves para a dispersão de suas sementes. Os pássaros utilizam os frutos na alimentação e após regurgitação ou defecação, as sementes liberadas ficam aderidas às plantas hospedeiras. Essa aderência é possível, pois a semente possui uma camada mucilaginosa ao redor (LEAL, 2006).

A espécie Tripodanthus acutifolius (Ruiz \& Pav.) Tiegh. é uma planta hemiparasita pertencente à família Loranthaceae, e pode ser considerada como uma das mais agressivas do grupo, principalmente pela diversidade de hospedeiros que afeta e a forma com que se prolifera pelo tronco, galhos e copa.

Até o momento já foram relatadas importantes atividades biológicas atribuídas a esta erva, como anti-inflamatória, analgésica, antipirética, antimicrobiana, antioxidante, hipotensora, diurética, hemostática e antitumoral, o que incita à busca de novas propriedades por meio de seus extratos e frações. 
A resistência a agentes antimicrobianos é grave e preocupante e requer não somente a pesquisa para o desenvolvimento de novas substâncias antimicrobianas, mas também o desenvolvimento de novas abordagens para o tratamento de infecções bacterianas.

Este estudo buscou avaliar o potencial antimicrobiano do extrato aquoso de Tripodanthus acutifolius frente a cepas de Staphylococcus aureus identificados quanto ao perfil de resistência e sensibilidade à meticilina.

\section{METODOLOGIA}

\subsection{Coleta e identificação do material vegetal}

A espécie Tripodanthus acutifolius (Figura 1) foi coletada do hospedeiro Ligustrum lucidum no município de Santa Cruz do Sul/RS, no mês de setembro do ano de 2010.

A identificação botânica foi realizada pela professora Marisa Terezinha Lopes Putzke, do Laboratório de Botânica da UNISC e sua exsicata foi depositada no Herbário do Instituto de Biociências da Universidade Federal do Rio Grande do Sul (UFRGS), sob o n $^{\circ}$ ICN 167796.

\section{Figura 1 - Folhas de Tripodanthus acutifolius}

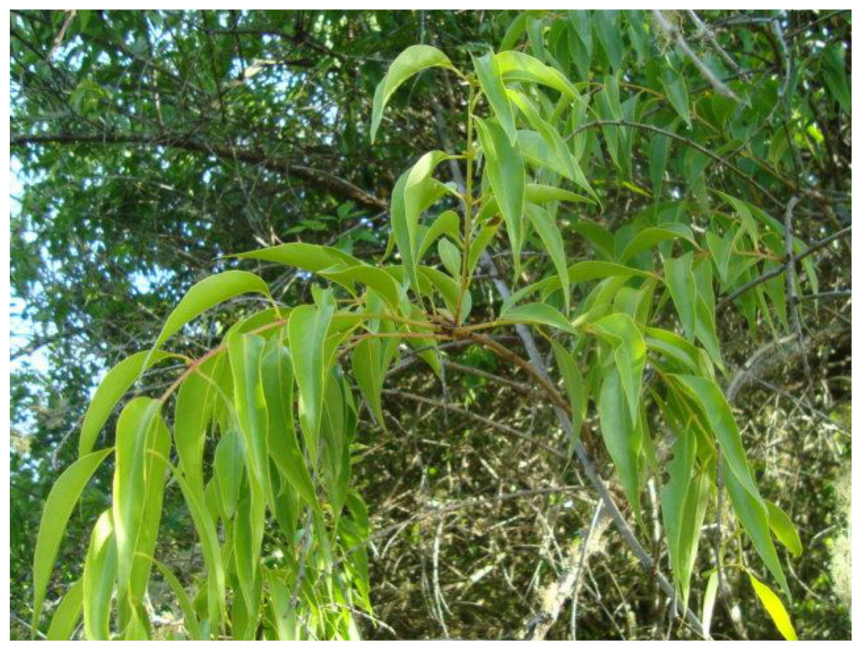

Após a coleta, as partes aéreas foram separadas e analisadas. Apenas as folhas íntegras e os caules finos, sem doenças, rasuras ou contaminados foram selecionados. Em seguida, as amostras foram lavadas em água corrente e secas em estufa de ar circulante (TECNAL®, modelo TE 394/3) por 48 horas à temperatura de $40^{\circ} \mathrm{C}$. Depois da secagem completa realizou-se a retirada dos caules, permanecendo como farmacógeno apenas as folhas inteiras e totalmente secas. Na sequência, as folhas foram moídas em moinho de facas (TECNAL®, modelo TE - 648) e armazenadas em frasco de vidro esterilizado e rotulado. 


\subsection{Obtenção do extrato aquoso de Tripodanthus acutifolius}

O extrato aquoso de Tripodanthus acutifolius foi preparado no Laboratório de Farmacognosia da Universidade de Santa Cruz do Sul (UNISC) da seguinte forma: as folhas previamente secas foram recobertas com água destilada por um período de 24 horas. Este extrato foi preparado pela técnica de maceração, sendo que após este período, o líquido extrativo foi filtrado e o solvente aquoso renovado por cinco dias consecutivos. O extrato obtido foi concentrado em evaporador rotatório (MARCONI®, modelo MA - 120) e posteriormente liofilizado (EDUARDS ${ }^{\circledR}$, modelo Modulyo 4K).

\subsection{Microrganismos indicadores}

Para os testes de atividade antimicrobiana foram utilizados isolados clínicos hospitalares de Staphylococcus aureus, sendo três resistentes à meticilina (MRSA) e três sensíveis à meticilina (MSSA), além de uma cepa de Staphylococcus aureus 25923 (padrão ATCC). As bactérias foram identificadas através das provas bioquímicas de catalase, coagulase e DNAse e por coloração de Gram.

\subsection{Manutenção dos microrganismos}

No momento do uso as cepas foram repicadas para caldo cérebro-coração (BHI), incubadas à $35^{\circ} \mathrm{C}$ e semeadas em placas de Ágar Triptona de Soja (TSA) para comprovação da pureza.

\subsection{Avaliação da atividade antimicrobiana}

Para a determinação da atividade antibacteriana de $T$. acutifolius foi utilizado o método de ágar-difusão em discos, descrito por Benitez et al. (2011), com modificações. Alíquotas de $10 \mu \mathrm{L}$ do extrato aquoso, reconstituído a uma concentração de $100 \mathrm{mg} / \mathrm{mL}$ e filtrados (filtros Millipore $\AA$, de 0,22 $\mu \mathrm{m}$ ), foram semeadas em discos de celulose $(6 \mathrm{~mm}$ ) previamente esterilizados e dispostos em placas de Ágar Müeller-Hinton (MH), previamente inoculadas com uma suspensão bacteriana contendo $1,5 \times 10^{8}$ células $/ \mathrm{mL}(0,5$ na escala de Mac Farland) de cada microrganismo-teste. Como controles positivos foram utilizados discos de antibióticos frente aos quais as bactérias apresentaram sensibilidade. Para tanto foi realizado um antibiograma prévio com os microrganismos-teste. Para o controle negativo foi utilizada solução salina estéril $(0,85 \%)$.

Antes de levar à estufa, as placas foram deixadas por uma hora em temperatura ambiente para difusão dos extratos. Em seguida as placas foram incubadas por $24 \mathrm{~h}$ a $35^{\circ} \mathrm{C}$, 
considerado esta temperatura ótima para o crescimento do microrganismo testado. Após o período de incubação a pretensa atividade antimicrobiana foi evidenciada pela formação de halos de inibição ao redor dos discos que foram medidos com o auxílio de um paquímetro.

As concentrações de $100 \mathrm{mg} / \mathrm{mL}, 75 \mathrm{mg} / \mathrm{mL}, 50 \mathrm{mg} / \mathrm{mL}, 25 \mathrm{mg} / \mathrm{mL}, 15 \mathrm{mg} / \mathrm{mL}, 10$ $\mathrm{mg} / \mathrm{mL}, 5 \mathrm{mg} / \mathrm{mL}, 2,5 \mathrm{mg} / \mathrm{mL}, 1,0 \mathrm{mg} / \mathrm{mL}$ e $0,5 \mathrm{mg} / \mathrm{mL}$ do extrato aquoso da planta foram testadas. Tais concentrações foram preparadas a partir de uma solução-estoque de $100 \mathrm{mg} / \mathrm{mL}$ a qual também foi testada, onde foram pesados 5 gramas do extrato da planta e diluídos em 50 $\mathrm{mL}$ de água destilada estéril. Todas as concentrações foram testadas em duplicata.

\section{RESULTADOS E DISCUSSÃO}

Os resultados dos ensaios de atividade antibacteriana do extrato aquoso de Tripodanthus acutifolius, testados pelo método de ágar-difusão em disco frente aos microrganismos-teste, encontram-se descritos na tabela 1 e referem-se à menor concentração de extrato, dentre todas as concentrações testadas, que foi capaz de inibir o crescimento das cepas de Staphylococcus aureus.

Tabela 1 - Menor concentração de extrato capaz de inibir o crescimento das bactérias testadas

\begin{tabular}{l|c}
\hline \multicolumn{1}{c|}{ Micro-organismos teste } & Extrato aquoso de Tripodanthus acutifolius \\
\hline S. aureus 055 A (MRSA) & $2,5 \mathrm{mg} / \mathrm{mL}$ \\
S. aureus 045 A (MRSA) & $15 \mathrm{mg} / \mathrm{mL}$ \\
S. aureus 039 A (MRSA) & $15 \mathrm{mg} / \mathrm{mL}$ \\
S. aureus 021 A (MSSA) & $15 \mathrm{mg} / \mathrm{mL}$ \\
S. aureus 018 A (MSSA) & $15 \mathrm{mg} / \mathrm{mL}$ \\
S. aureus 017 A (MSSA) & $2,5 \mathrm{mg} / \mathrm{mL}$ \\
S. aureus 25923 (ATCC) & $0,5 \mathrm{mg} / \mathrm{mL}$ \\
\hline
\end{tabular}

MRSA: Staphylococcus aureus Resistente à Meticilina; MSSA: Staphylococcus aureus Sensível à Meticilina; ATCC: American Type Culture Collection.

Através da técnica de ágar-difusão em disco (BAUER et al., 1966) pode-se observar que todas as cepas de Staphylococcus aureus testadas neste estudo foram sensíveis à ação do extrato da planta diferentes diluições pela formação de zonas de inibição do crescimento microbiano de diâmetros variados.

De acordo com Black (2002) a atividade antimicrobiana nesta técnica ocorre através da difusão do agente quimioterápico em todas as direções do ágar durante a incubação. As cepas que se mostraram mais sensíveis ao extrato de Tripodanthus acutifolius foram 
Staphylococcus aureus 25923 (padrão AATC), 055 A (MRSA) e 017 A (MSSA), tendo seu crescimento inibido até a concentração de $0,5 \mathrm{mg} / \mathrm{mL}, 2,5 \mathrm{mg} / \mathrm{mL}$ e $2,5 \mathrm{mg} / \mathrm{mL}$ respectivamente, enquanto o crescimento de Staphylococcus aureus 045 A (MRSA), 039 A (MRSA), 021 A (MSSA) e 018 A (MSSA) foi inibido até a concentração de $15 \mathrm{mg} / \mathrm{mL}$.

A bactéria $S$. aureus é um coco Gram-positivo, anaeróbio facultativo, mas que cresce melhor em ambientes aeróbios. Esse microrganismo é capaz de produzir muitas toxinas que contribuem para a sua patogenicidade, o que a torna mais hábil para invadir o corpo e danificar os tecidos (TORTORA; FUNKE; CASE, 2003).

Foi descoberta uma droga do grupo das penicilinas, denominada meticilina, que não era suscetível à ação das betalactamases. Logo em seguida à meticilina vieram as cefalosporinas. Porém, no início da década de 1970, começaram a aparecer, com muita rapidez, cepas de $S$. aureus com resistência à meticilina identificadas pela sigla MRSA ( $S$. aureus resistente à meticilina), também resistentes aos demais betalactâmicos (cefalosporinas e outros).

De acordo com Stratton (2000), S. aureus meticilina resistente é o principal patógeno nosocomial em infecções hospitalares e é responsável por inativar a ação de vários antibióticos, tornando a multirresistência um grande problema de saúde pública. Em razão ao grande aumento da resistência de microrganismos patogênicos a múltiplas drogas, devido ao uso indiscriminado de antimicrobianos, surge a necessidade para a procura de novas alternativas terapêuticas (NOVAIS et al., 2003; ANTUNES et al., 2006; OLIVEIRA et al., 2007).

Considerando o grande abuso do uso de antibióticos tradicionais e o rápido aumento da resistência de bactérias a estes, há um significativo interesse em pesquisas com plantas a fim de verificar o potencial antimicrobiano de seus extratos (PEREIRA et al., 2010). No presente estudo, tanto as cepas de Staphylococcus aureus resistentes à meticilina (MRSA) quanto as cepas sensíveis à meticilina (MSSA) e a cepa padrão ATCC apresentaram sensibilidade a várias concentrações do extrato aquoso da erva-de-passarinho (Figuras 2 a 5).

Em um estudo realizado por Adwan e Mhanna (2008), os extratos das plantas Psidium guajava, Rosmarinus officinalis, Salvia fruticosa, Majorana syriaca, Ocimum basilucum, Syzygium aromaticum, Laurus nobilis e Rosa damascena provocaram a redução das CIM de vários antibióticos frente a isolados de S. aureus resistentes (MRSA) e S. aureus sensíveis (MSSA) provenientes de processos clínicos humanos. No entanto, a redução foi maior frente aos isolados resistentes. Neste trabalho, constatou-se que a maior atividade antimicrobiana dos extratos da planta ocorreu frente à cepa padrão ATCC de S. aureus. 
No Brasil, as pesquisas sobre substâncias antimicrobianas de origem vegetal iniciaram-se com Cardoso e Santos (1948), que avaliaram extratos de 100 diferentes plantas indicadas em terapêutica popular como anti-inflamatórios ou cicatrizantes/antimicrobianos. Destas plantas, cinco extratos apresentaram atividade contra S. aureus, Escherichia coli e Proteus X-19. No presente estudo podemos sugerir, a partir dos resultados, uma provável atividade antibacteriana do extrato aquoso de Tripodanthus acutifolius dirigida a cepas de $S$. aureus resistentes e sensíveis à meticilina.

A investigação sobre produtos naturais com atividade antimicrobiana contra MRSA aumentou significativamente nos últimos anos. Machado et al. (2003), estudando 14 extratos de plantas usadas tradicionalmente no Brasil para tratamento de doenças infecciosas, verificaram que Punica granatum (romã) inibiu linhagens de $S$. aureus sensíveis e resistentes à meticilina, concluindo que esta substância apresenta potencial como agente no tratamento de infecções causadas por MRSA.

Comparando-se a atividade do extrato da erva-de-passarinho com a atividade antimicrobiana de diversos extratos vegetais, incluindo o cajueiro testado sobre $S$. aureus, os resultados mostraram que para $80 \%$ das linhagens o extrato foi potencialmente ativo apresentando halos de inibição superiores a 15 mm de diâmetro (CEBALHOS et al., 1993). Nesta investigação, o extrato de Tripodanthus acutifolius inibiu o crescimento das cepas de $S$. aureus, apresentando halos de inibição que chegaram até $24 \mathrm{~mm}$ de diâmetro, como pode ser visto na Tabela 2.

Tabela 2 - Atividade antibacteriana do extrato aquoso (EA) de Tripodanthus acutifolius frente aos microrganismos testados

\begin{tabular}{|l|c|c|}
\hline \multicolumn{1}{|c|}{ Micro-organismos teste } & $\begin{array}{c}\text { Concentrações do EA } \\
(\mathbf{m g} / \mathbf{m L})\end{array}$ & Variações dos halos (mm) \\
\hline S. aureus 055 A (MRSA) & $100-2,5$ & $21,42-9,40$ \\
\hline S. aureus 045 A (MRSA) & $100-15$ & $18,56-9,70$ \\
\hline S. aureus 039 A (MRSA) & $100-15$ & $18,92-10,68$ \\
\hline S. aureus 021 A (MSSA) & $100-15$ & $19,68-12,90$ \\
\hline S. aureus 018 A (MSSA) & $100-15$ & $21,64-14,95$ \\
\hline S. aureus 017 A (MSSA) & $100-2,5$ & $24,67-12,00$ \\
\hline S. aureus 25923 (ATCC) & $100-0,5$ & $24,73-9,24$ \\
\hline
\end{tabular}

MRSA: Staphylococcus aureus Resistente à Meticilina; MSSA: Staphylococcus aureus Sensível à Meticilina; ATCC: American Type Culture Collection; EA: Extrato aquoso. 
No estudo de Santos e Alves (2012) as cepas de S. aureus, testadas frente a onze diferentes extratos vegetais, também tiveram inibição do crescimento. Dessa forma pode-se intuir que extratos vegetais e suas frações, em diferentes concentrações, podem ser capazes de afetar o crescimento desta bactéria, já que em variados estudos mostraram algum grau de sensibilidade.

Figura 2 - $S$. aureus $(045 \mathrm{~A})$ resistente à meticilina inibida pelo extrato aquoso de $T$. acutifolius até $15 \mathrm{mg} / \mathrm{mL}(\mathrm{A})$; S. aureus (039 A) resistente à meticilina inibida pelo extrato aquoso de $T$. acutifolius até $15 \mathrm{mg} / \mathrm{mL}$ (B)
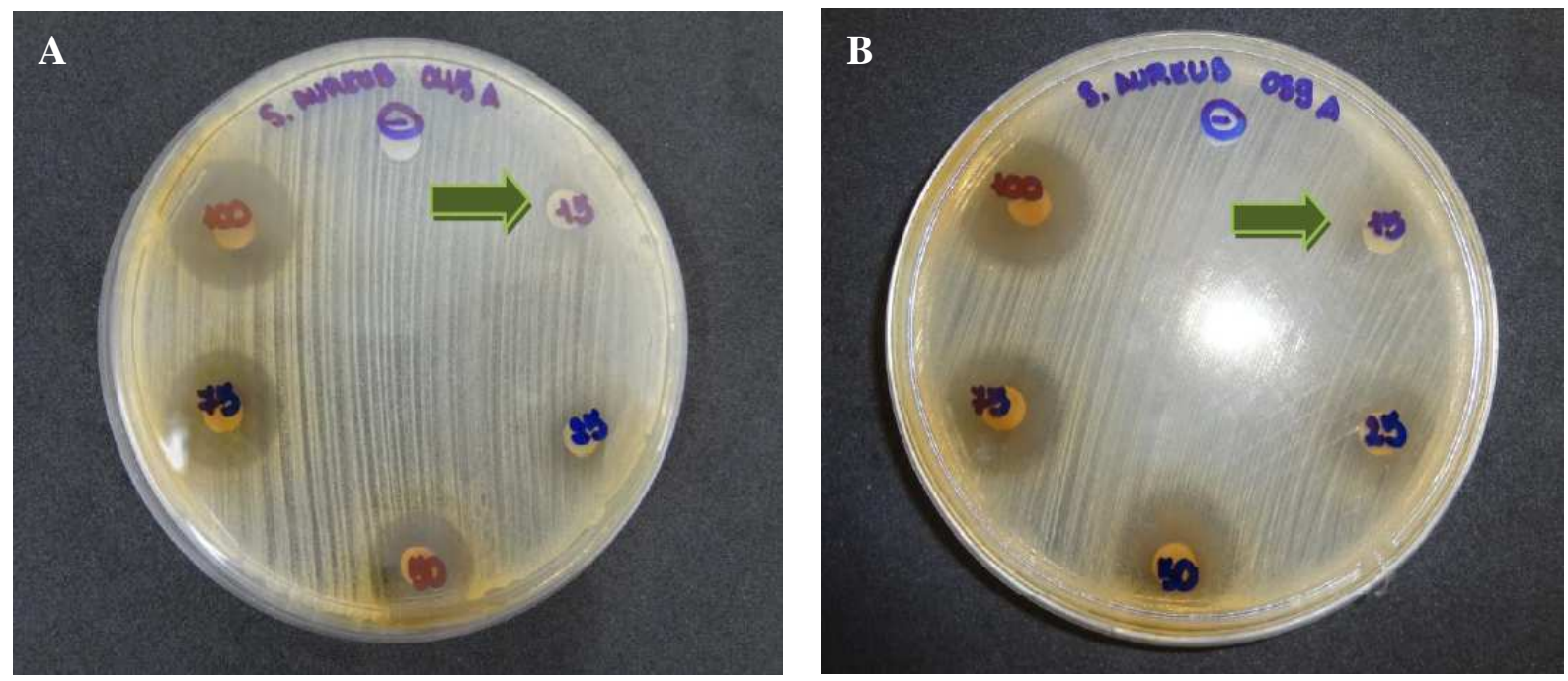

Figura 3 - S. aureus $(055 \mathrm{~A})$ resistente à meticilina inibida pelo extrato aquoso de $T$. acutifolius (A); S. aureus (055 A) resistente à meticilina inibida pelo extrato aquoso de $T$. acutifolius até $2,5 \mathrm{mg} / \mathrm{mL}$ (B)
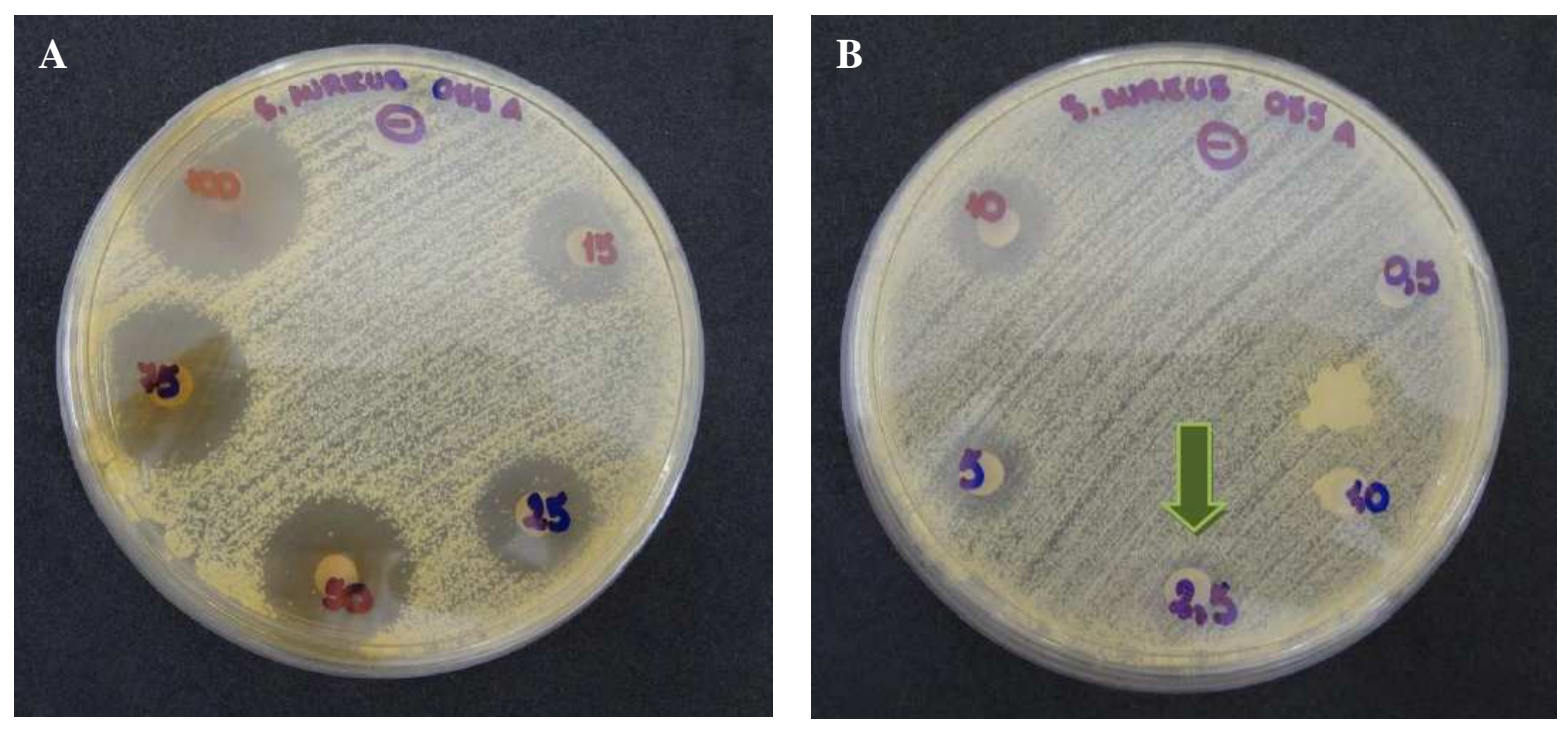
Figura 4 - S. aureus (017 A) sensível à meticilina inibida pelo extrato aquoso de $T$. acutifolius (A); $S$. aureus (017 A) sensível à meticilina inibida pelo extrato aquoso de $T$. acutifolius até $2,5 \mathrm{mg} / \mathrm{mL}$ (B)
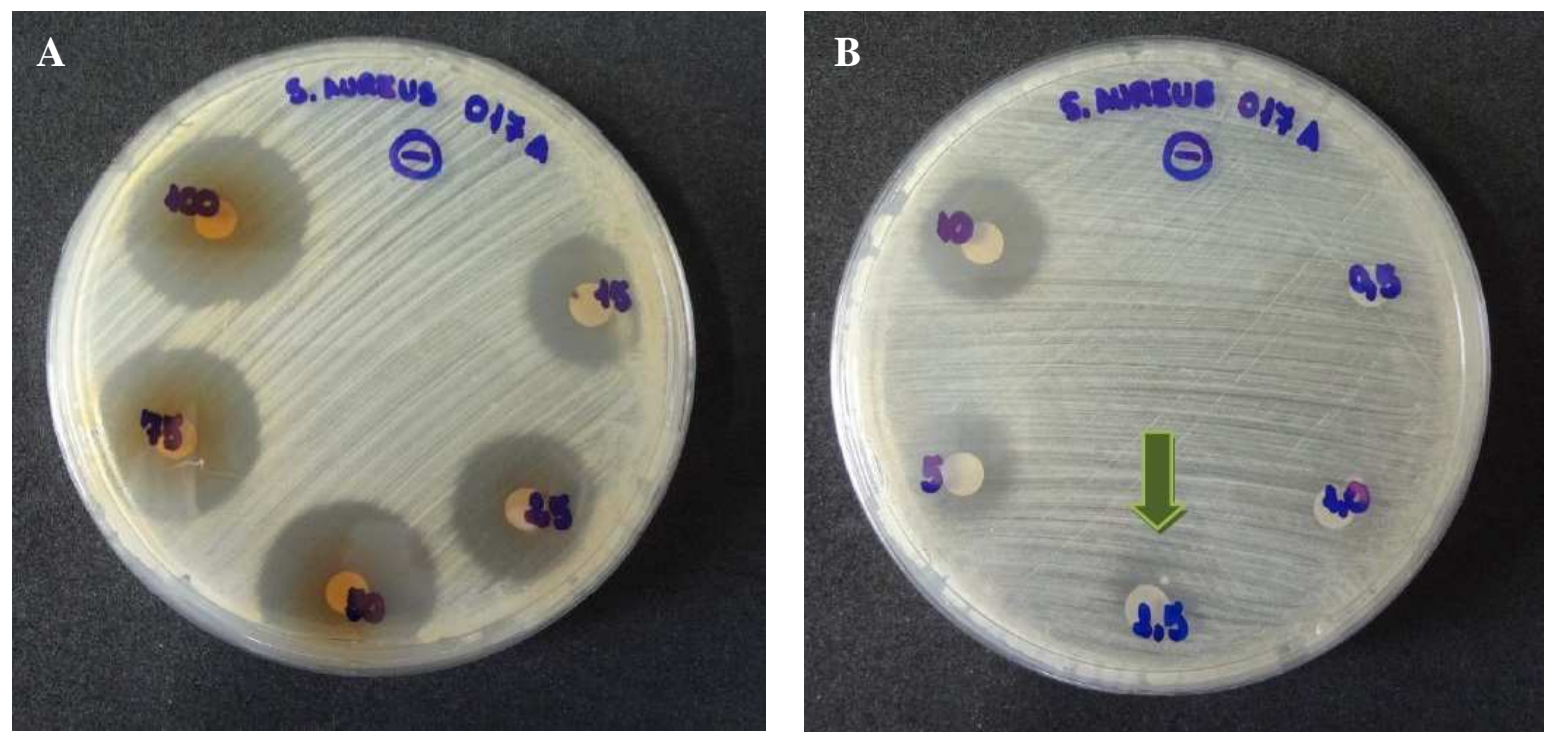

Figura 5 - S. aureus (018 A) sensível à meticilina inibida pelo extrato aquoso de $T$. acutifolius até $15 \mathrm{mg} / \mathrm{mL}(\mathrm{A}) ;$ S. aureus (021 A) sensível à meticilina inibida pelo extrato aquoso de $T$. acutifolius até $15 \mathrm{mg} / \mathrm{mL}$ (B)
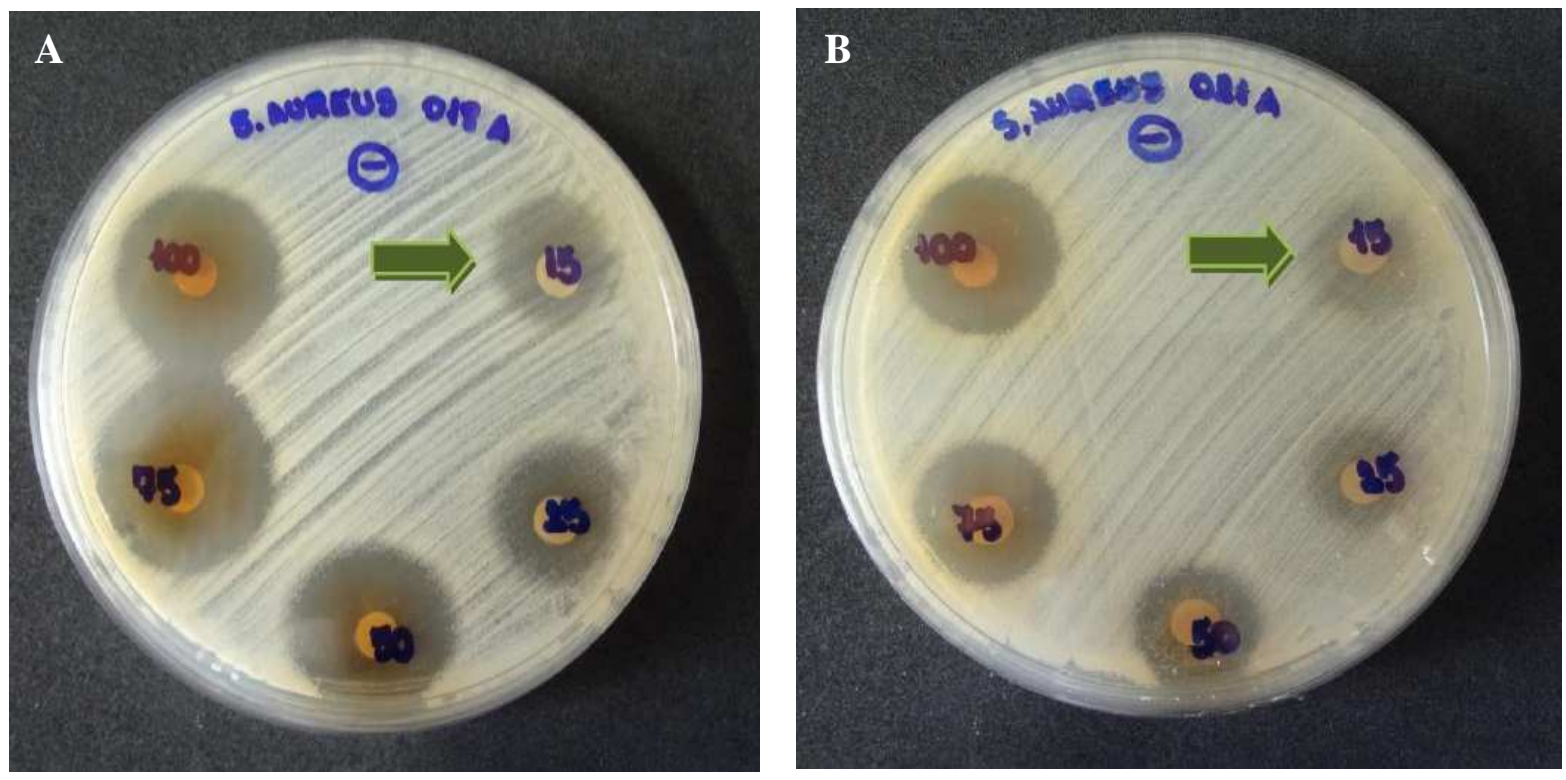
O Método de Ágar-difusão apresenta como vantagens a possibilidade de testar contra um único microrganismo até seis extratos por placa, com a utilização de pequeno volume de amostra, além de ser considerada uma metodologia conveniente devido a sua simplicidade e baixo custo. O método por difusão não é apropriado para análise de compostos que não se difundem facilmente no ágar, sendo este parâmetro considerado a principal desvantagem do ensaio (OSTROSKY et al., 2008).

A atividade frente à $S$. aureus foi verificada para o extrato metanólico das folhas e cascas de D. parviflora e D. pseudo sisso pelo método de ágar-difusão em disco na concentração de $50 \mathrm{mg} / \mathrm{mL}$ (CHUNG et al., 2004). No presente trabalho, todas as cepas de $S$. aureus também foram inibidas pela concentração de $50 \mathrm{mg} / \mathrm{mL}$ do extrato aquoso de Tripodanthus acutifolius.

No estudo conduzido por Tadeg et al. (2005), extratos hidroalcoólicos de 8 espécies de plantas medicinais usadas no tratamento de desordens de pele foram avaliados quanto à atividade antimicrobiana em relação a bactérias e fungos conhecidos por causarem diferentes tipos de infecções cutâneas. A atividade antimicrobiana foi determinada utilizando o método de ágar-difusão nas concentrações de 100, 50 e $25 \mathrm{mg} / \mathrm{mL}$ para os extratos brutos e 25 e 5 $\mathrm{mg} / \mathrm{mL}$ para as frações. Neste estudo a cepa de S. aureus 25923 (padrão ATCC) teve o seu crescimento inibido pela menor concentração do extrato aquoso $(0,5 \mathrm{mg} / \mathrm{mL})$ e ambas as cepas de S. aureus resistentes à meticilina (MRSA) e as sensíveis à meticilina (MSSA) foram inibidas pelas concentrações de 15 e $2,5 \mathrm{mg} / \mathrm{mL}$.

A atividade frente à $S$. aureus foi verificada para o extrato metanólico das folhas e cascas de D. parviflora e D. pseudo sisso pelo método de ágar-difusão em disco na concentração de $50 \mathrm{mg} / \mathrm{mL}$ (CHUNG et al., 2004). No presente trabalho, todas as cepas de $S$. aureus também foram inibidas pela concentração de $50 \mathrm{mg} / \mathrm{mL}$ do extrato aquoso de Tripodanthus acutifolius.

A identificação de constituintes químicos com potencial farmacológico para comprovar as propriedades medicinais da erva de passarinho tem sido alvo de inúmeras investigações (RICCO et al., 2008; SOBERÓN et al., 2010a; SOBERÓN et al., 2010b). No estudo de Grüner et al. (2012) foi realizada a análise fitoquímica a partir dos extratos aquoso e metanólico das folhas da planta Tripodanthus acutifolius, utilizada neste trabalho, e foi possível evidenciar a presença de flavonóides, taninos e compostos antracênicos. 


\section{CONCLUSÕES}

A partir dos resultados obtidos neste estudo foi possível concluir que todos os isolados de $S$. aureus, tanto os resistentes quanto os sensíveis à meticilina, tiveram seu crescimento afetado pelo extrato aquoso de Tripodanthus acutifolius. A cepa de $S$. aureus 25923 (padrão ATCC) teve o seu crescimento inibido pela menor concentração do extrato aquoso $(0,5 \mathrm{mg} / \mathrm{mL})$, enquanto que ambas as cepas de MRSA e MSSA foram inibidas pelas concentrações de 15 e 2,5 mg/mL. O método de ágar-difusão, utilizado nesta pesquisa, mostrou-se eficiente uma vez que foi capaz de detectar a inibição do crescimento das cepas de $S$. aureus em concentrações menores de $2,5 \mathrm{mg} / \mathrm{mL}$ e $0,5 \mathrm{mg} / \mathrm{mL}$, apresentando halos de inibição de até $24 \mathrm{~mm}$ diâmetro. A atividade antibacteriana exibida pela "erva-de-passarinho" frente às cepas MRSA e MSSA torna esta planta interessante para estudos complementares de isolamento e identificação dos seus componentes ativos e de possíveis modificações químicas capazes de aumentar seu potencial antimicrobiano para que possa ser usada como medicamento fitoterápico no tratamento de múltiplas infecções. Este trabalho forneceu evidências científicas sobre a atividade farmacológica atribuída à Tripodanthus acutifolius e contribuiu para validar seu uso a partir das infusões das folhas da planta pela medicina popular.

\section{REFERÊNCIAS}

ADWAN, D. \& MHANNA, M. Synergistic Effects of Plant Extracts and Antibiotics on Staphylococcus aureus. Strains Isolated from Clinical Specimens. Middle-East Journal of Scientific Research, v. 3, n. 3, p. 134-139, 2008.

ANTUNES, R. M. P.; LIMA, E. O.; PEREIRA, M. S. V.; CAMARA, C. A.; ARRUDA, T. A.; CATÃO, R. M. R.; BARBOSA, T. P.; NUNES, X. P.; DIAS, C. S.; SILVA, T. M. S. Atividade antimicrobiana "in vitro" e determinação da concentração inibitória mínina (CIM) de fitoconstituintes e produtos sintéticos sobre bactérias e fungos leveduriformes. Revista Brasileira de Farmacognosia, v. 16, p. 517-524, 2006.

BAUER, A. W.; KIRBY, W. M. M.; SHERRIS, J. C.; TURCK, M. Antibiotic susceptibity testing by a standardized single disk method. American Journal of Clinical Pathology, v. 4, p. 493-496, 1966.

BENITEZ, L. B. et al. Antimicrobial activity of Bacillus amyloliquefaciens LBM 5006 is enhanced in the presence of Escherichia coli. Current Microbiology, v. 62, p. 1017-1022, 2011.

BLACK, J. G. Microbiologia: fundamentos e perspectivas. 4 ed. Rio de Janeiro: Guanabara Koogan, 2002. 
CARDOSO, H. T.; SANTOS, M. L. Estudos sobre a presença de antibióticos nos vegetais. Bras Med, v. 62, p. 67-70, 1948.

CEBAlHOS, B. S. O.; URTIGA, R. F.; BARBOSA, R. C. S. B. C.; LIMA, E. O. Atividade Antimicrobiana de produtos naturais sobre Staphylococcus aureus e Pseudomonas aeruginosa isoladas de águas recreacionais. Revista Brasileira de Farmacognosia, v. 74, p. 4-6, 1993.

CHUNG, P. Y.; CHUNG, L. Y., NGEOW, Y. F.; GOH, S. H.; IMIYABIR, Z. Antimicrobial Activities of Malaysian Plant Species. Pharmaceutical Biology, v. 42, n. 4-5, p. 292-300, 2004.

GRÜNER, J. M.; SOUZA, T. K.; BENITEZ, L. B.; SILVA, C. M. Análise do perfil fitoquímico de Tripodanthus acutifolius (Ruiz \& Pavón) Tieghem, Loranthaceae. Revista Jovens Pesquisadores, n. 1, p. 9-17, 2012.

LEAL, L.; BUJOKAS, W. M.; BIONDI, D. Análise da infestação de erva-de-passarinho na arborização de ruas de Curitiba - PR. Floresta, v. 36, n. 3, 2006.

MACHADO, T. B.; PINTO, A. V.; PINTO, M. C. F. R.; LEAL, I. C. R.; SILVA, M. G.; AMARAL, A. C. F.; KUSTER, R. M.; NETTO-DOSSANTOS, K. R. In vitro activity of Brazilian medicinal plants, naturally occurring naphthoquinones and their analogues, against methicillin-resistant Staphylococcus aureus. International Journal of Antimicrobial Agents, v. 21, p. 279-284, 2003.

NOVAIS, T. S.; COSTA, J. F. O.; DAVID, J. P. L.; DAVID, J. M.; QUEIROZ, L. P.; FRANÇA, F.; GIULIETTI, A. M.; SOARES, M. B. P.; SANTOS, R. R. Atividade antibacteriana em alguns extratos de vegetais do semi-árido brasileiro. Revista Brasileira de Farmacognosia, v. 13 (Supl. 2), p. 5-8, 2003.

NUNES, L. G. Prospecção fitoquímica e avaliação de mutagenicidade in vitro de três espécies vegetais: Strychnos pseudoquina A. St.-Hil., Coutarea hexandra (Jacq.) K. Shum e Bathysa cuspidata (A. St.-Hil.) Hook. Viçosa: Universidade Federal de Viçosa, 2008.

OLIVEIRA, R. A. G.; LIMA, E. O.; SOUZA, E. L.; VIEIRA, W. L.; FREIRE, K. R. L.; TRAJANO, V. N.; LIMA, I. O.; SILVA-FILHO, R. N. Interference of Plectranthus amboinicus (Lour.) Spreng essential oil on the anti-Candida activity of some clinically used antifungals. Revista Brasileira de Farmacognosia, v. 17, p. 186-190, 2007.

OSTROSKY, E. A. et al. Métodos para avaliação da atividade antimicrobiana e determinação da concentração mínima inibitória (CMI) de plantas medicinais. Revista Brasileira de Farmacognosia, v. 18, n. 2, p. 301-307, 2008.

PEREIRA, M. do S. V. et al. Efeitos antimicrobianos e genéticos de extratos vegetais sobre plasmídios de resistência a antibióticos em microrganismos de origem bovina. Revista de Biologia e Farmácia, v. 4, n. 1, p. 26-31, 2010.

RICCO, R. A. et al. Modificación em el perfil de polifenoles de Tripodanthus acutifolius (Ruiz et Pav.) Tiegh. (Loranthaceae) infectado por "cochinilla" (Homoptera - Lecanidae). Latin American Journal of Pharmacy, v. 27, n. 2, p. 258-62, 2008. 
ROTTA, E. Autotrofia em Tripodanthus acutifolius (Ruiz \& Pav.) Thiegh. (erva-depassarinho) - um registro. Colombo: 2004.

SANTOS, S. de J. D.; ALVES, F. Análise comparativa da ação de extratos de plantas com atividade antimicrobiana (in vitro) sobre cepas de Staphylococcus aureus. Periódico Científico do Núcleo de Biociências, v. 2, n. 4, p. 2302012.

SOBERÓN, J. R. et al. Antibacterial activity of plant extracts from northwestern Argentina. Journal of Applied Microbiology, v. 102, p. 1450-1461, 2007.

SOBERÓN, R. J. et al. Free radical scavenging activities and inhibition of inflammatory enzymes of phenolics isolated from Tripodanthus acutifolius. Journal of Ethnopharmacology, v. 130, p. 329-333, 2010a.

SOBERÓN, R. J. et al. Study of antiinflammatory activity of metabolites isolated from Tripodanthus acutifolius. Molecular Medicinal Chemistry, v. 21, p. 88-90, 2010b.

STRATTON, C. W. Nuances in antimicrobial susceptibility testing for resistant gram-positive organisms. Antimicrobics and Infectious Diseases Newsletter, v. 18, p. 57-64, 2000.

TADEG, H.; MOHAMMED, E.; ASRES, K.; GEBRE-MARIAN, T. Antimicrobial activities of some selected traditional Ethiopian medicinal plants used in treatment of skin disorders. $J$ Ethnopharmacol, v. 100, p. 168-175, 2005.

TORTORA, G. J; FUNKE, B. R; CASE, C. L. Microbiologia. São Paulo: Artmed Editora S. A., 2003. 